\title{
P 266 A SPECIALIST PALLIATIVE CARE SERVICE FOR PARKINSON'S DISEASE: REVIEW OF END OF LIFE OUTCOMES
}

Debi Adams, ${ }^{1}$ Edward Richfield, ${ }^{2,3}$ Miriam Johnson, ${ }^{1,3}$ Edward Jones, ${ }^{2}$ Colin Campbell'. 'Saint Catherine's Hospice, Scarborough, UK; ${ }^{2}$ Department for Elderly Medicine, Scarborough General Hospital, York NHS Trust, Scarborough, UK; ${ }^{3}$ Hull York Medical School, The University of Hull, Hull, UK

\subsection{6/bmjspcare-2014-000654.307}

Background National guidance supports the need for specialist palliative care (SPC) in Parkinson's disease (PD). Access to SPC remains poor and evidence to support palliative care strategies in PD is scarce.

Innovation The Scarborough PD SPC service is a collaboration between St Catherine's Hospice and the movement disorder clinic at Scarborough General Hospital. We report on SPC service utilisation and outcomes.

Evaluation The case-notes of 47 PD patients consecutively referred to SPC (Nov 2010 to May 2013) were retrospectively reviewed (34 male; mean age 77 yrs; range 53 to 99). Hospice services/interventions received included: palliative neurology CNS support (100\%); future care planning (60\%); day hospice attendance (26\%); hospice admission for respite care (21\%), symptom management (21\%) and care of dying (14\%). 23 patients died. Average time to death from referral was 194 days (range 2-753 days) and from diagnosis was 10 years (range 2-25 years). Place of death was known for 22/23 patients: acute hospital bed (13\%); hospice (26\%); home (13\%); care home (34\%) and community palliative care bed (8\%). Dying was anticipated in $91 \%$ of patients (21/23), all of whom had a DNACPR order and an advance care plan or best interest decision. Administration of anti-Parkinson's medication in the dying phase was problematic and rigidity a common symptom, although rarely with signs of distress. Average time from identification of dying to death was 8.3 days (range 2-23 days) compared with 29 hours across all diseases (MCPCI 2012).

Conclusions People with PD and palliative care needs can benefit from referral to SPC. Rates of death in hospital were reduced and deaths at home/hospice increased compared with UK data (Snell K 2009). The dying phase, although prolonged, can be managed well and choice and comfort at the very end of life are achievable. 